\title{
Providing Proximity Safety Alerts to Workers on Construction Sites Using Bluetooth Low Energy RTLS
}

\author{
Yusheng Huang ${ }^{1}$, Amin Hammad ${ }^{2}$ and Zhenhua Zhu ${ }^{3}$ \\ 1 Concordia University, Montreal, Canada \\ 2 Concordia University, Montreal, Canada (corresponding author) \\ 3 University of Wisconsin-Madison, Wisconsin, USA
}

\begin{abstract}
Struck-by equipment is one of the main reasons of accidents on construction sites. In order to improve construction safety, previous research proposed using Real-Time Location Systems (RTLS) to track the location of workers and equipment on construction sites. However, using ultra-wideband RTLS on large sites is difficult because it needs many timing cables to synchronize the data of the sensors surrounding the site. Furthermore, providing safety alerts to workers within dangerous proximity to equipment has not been addressed in previous research. Instead, the alerts were sent only to the safety manager. This paper aims to develop a method for providing proximity safety alerts to workers on construction sites using Bluetooth Low Energy (BLE) RTLS. BLE RTLS can provide acceptable accuracy coupled with large coverage and without the need of timing cables. In addition, with the support of two-way communications between the tags and sensors, it is possible to provide vibro-tactile alerts to the workers using wristbands. A prototype system is developed to filter the location data and remove outliers using averaging over time and averaging over tags of the objects. A case study is applied on a construction site to demonstrate the feasibility and performance of the proposed method.
\end{abstract}

(c) 2020 The Authors. Published by Budapest University of Technology and Economics \& Diamond Congress Ltd Peer-review under responsibility of the Scientific Committee of the Creative Construction Conference 2020.

Keywords: Bluetooth Low Energy (BLE), Real-time Location System (RTLS), construction safety, wristband, vibro-tactile alert

\section{Introduction}

Struck-by equipment is one of the main reasons of accidents on construction sites [1]. In order to improve construction safety, previous research proposed using Real-Time Location Systems (RTLS) to track the locations of workers and equipment on construction sites. However, it is important to find the balance between positioning quality and cost of the RTLS. For methods using the low-cost radio-frequency identification (RFID), the accuracy is too low for safety management on construction sites [2, 3]. Technologies such as ultra-wideband (UWB) and the surveying-level Global Positioning System (GPS), on the other hand, are accurate but expensive, which reduces their applicability in construction sites. Moreover, UWB RTLS requires timing cables to synchronize the data of the sensors surrounding the site [4, 5], which is another difficulty for construction projects since more cabling means more cost. In addition, in previous research, alerts were sent only to the safety manager, or risk events were recorded in reports which can be reviewed by the safety manager on a daily or weekly basis. To prevent struct-by accidents more effectively, a method that can warn workers in near real-time is needed.

This paper aims to develop a method with less cabling using Bluetooth Low Energy (BLE) RTLS based on the angle of arrival (AOA) technique, which provides sub-meter positioning accuracy with a relatively lower cost compared to UWB [6], to provide near real-time proximity safety alerts to workers on a construction site. The research has three objectives: (1) Testing the feasibility of using a wireless scheme of RTLS on 
construction sites; (2) Developing a method which effectively detects proximities in near real-time; and (3) Testing vibro-tactile alert patterns sent to workers which can be easily perceived.

\section{Purposed method}

The method focuses on data processing to define proximity events, and generating near real-time alerts and weekly proximity reports. First, the BLE system setting is configured to meet the requirements according to the constraints. Then, data processing is discussed to illustrate how to define the proximity. At last, the method of generating near real-time alerts and events recording is presented.

\subsection{BLE RTLS setting for meeting requirements}

Five requirements are identified for BLE RTLS in order to get good positioning performance: (1) Field of view (FOV) of sensors: FOV of sensors is the most important factor affecting the performance of the system. To guarantee good performance, the sensors should be installed where they have line of sight to as many tags as possible. (2) Data handling capacity of sensors: The RTLS sensors have limited capacity for handling tags data, which depends on the specific type of the sensors. For the BLE RTLS used in this project, the maximum capacity that the sensors can handle is 250 packets/s [6]. (3) Network environment and power supply: The sensors should be connected to the same network of the server computer. One solution to reduce the need for long ethernet cables is to build a wireless network environment using antennas as shown in Figure 1. (4) Visibility of tags: Although setting sensor at a high position can help increase the visibility of tags, there are occlusions which are inevitable. Since the construction site is a dynamic place, tags can be occluded for short periods by different construction resources. To address this issue, several tags are attached on the same object, so that even if data of one tag is missing, the position of the object can still be calculated by using data from the other tags. (5) Reflective materials: Signals can be reflected by the metallic surfaces on the construction site. Since the BLE RTLS uses AOA to calculate the position of tags, reflections of the signals will generate more errors in the results.

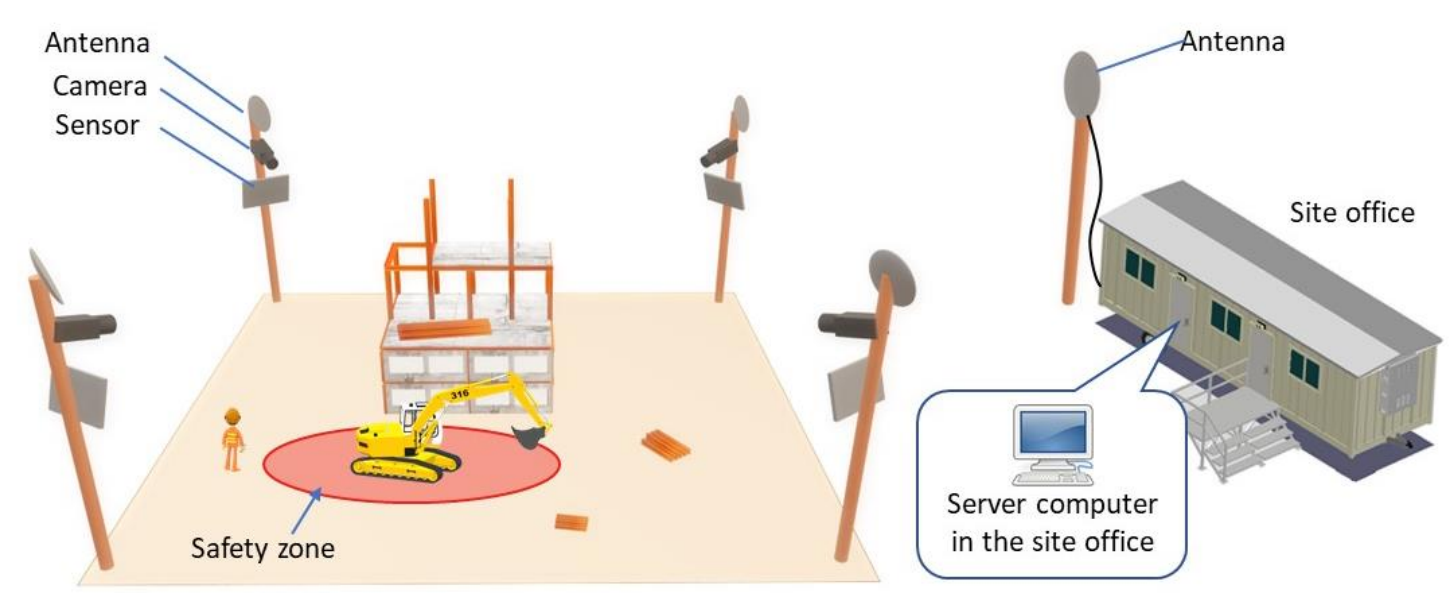

Figure 6. Using Antenna to Connect Sensors to Server in Site Office

\subsection{Data processing}

Although filters are embedded in most of RTLS systems, the smoothed data still have many errors. Therefore, extra processing is applied to reduce errors. The steps of data processing are shown in Figure 7: (1) Identifying the tags attached to the same object; (2) Averaging position data of the same tag over a period of time $d_{t}$ to get position at time $T_{i}$; (3) Estimating the pose and the velocity of an object based on the known geometric relationship between tags on that object; (4) If the data of an object at time $T_{i}$ is missing, the position of the object at time $T_{i}$ is calculated using extrapolation. 


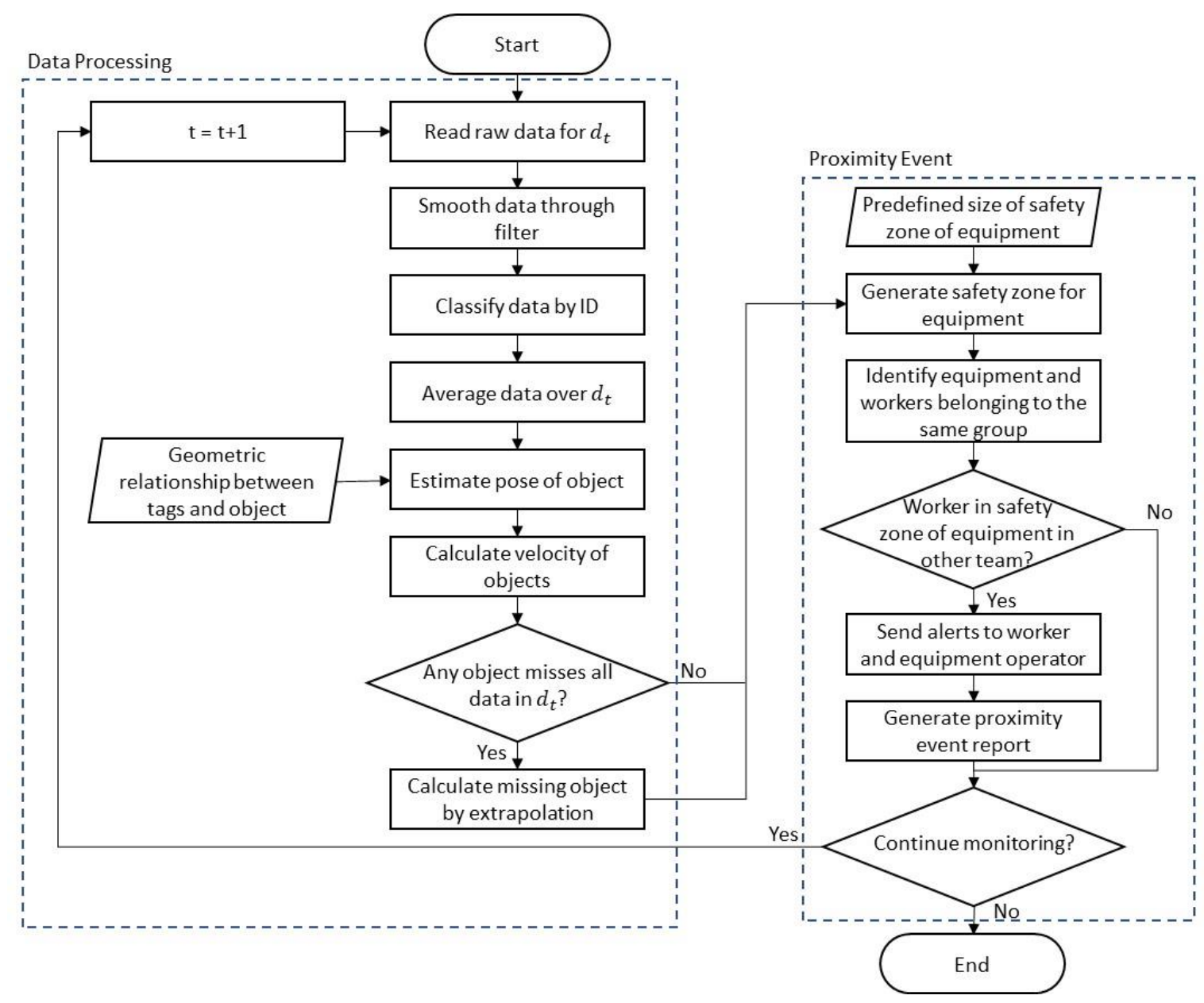

Figure 7. Data Processing and Proximity Event

\subsection{Proximity events}

Using the positions of workers and heavy equipment, proximity events can be detected. The size of the safety zone for each equipment is selected at the planning phase. In this paper, safety zones are designed to have a circular shape with a radius corresponding to the size of equipment as shown in Figure 6 . After getting the position of equipment, dynamic safety zones are generated. In the proximity detection step, whenever a worker enters the safety zone of an equipment not belonging to the same group, this is considered as a proximity event. In this case, alerts are sent to the worker and equipment operator through vibro-tactile signals. Information of this event, including the time, ID and position of involved the worker and equipment, are also recorded in order to generate a daily/weekly safety report.

\subsection{Generating alerts}

Three tactile signals are designed to be sent to workers and equipment operators. Based on the results of Saket et al. [7], the user's feelings and perceptions are different for different vibration patterns. With the same vibration strength, a rapid pattern produces a more urgent feeling to the user. Three vibration patterns are designed to represent three different cases of proximity. The three patterns are shown in Figure 8 . When a worker enters a safety zone, Pattern 1 is generated. If the worker continues approaching the equipment, then Pattern 2 is generated. If the worker enters a very dangerous zone (i.e. very close to the equipment), Pattern 3 is generated.

\begin{tabular}{l}
\begin{tabular}{|c|c|c|c|c|c|c|c|}
\hline \multicolumn{2}{|c|}{ on } & \multicolumn{2}{|c|}{ off } & \multicolumn{2}{c|}{ on } & \multicolumn{2}{c|}{ off } \\
\hline on & off & on & off & on & off & on & off \\
\hline \multicolumn{8}{|c|}{ on } \\
\hline
\end{tabular} \\
\hline
\end{tabular}

(1) Long on, long off

(2) Short on, short off

Figure 8. Vibration Patterns 


\section{Case study}

In the case study, tests are conducted to evaluate the feasibility of the proposed method on a construction site of an electric substation. The area of the construction site is about $110 \mathrm{~m} \times 70 \mathrm{~m}$. The RTLS sensors and antennas are installed on four poles at the corners of the construction site. Another antenna was installed on the pole near the site office. The site office is located at a distance of more than $100 \mathrm{~m}$ away from the construction site.

\subsection{RTLS installation and setup}

The BLE RTLS used in the case study is Quuppa [6]. The sensors are of the model LD7L, which is suitable for outdoor positioning and can achieve sub-meter accuracy within the range of $150 \mathrm{~m}$. A total of nine sensors are installed on the four poles. Antennas are installed at the top of poles and sensors are connected to the antenna on the same pole. On each pole, a camera is also installed to capture the video of the site. The videos are used to visually compare with the results from RTLS. The positions of sensors are measured using a total station. The setting of the RTLS is configured to optimizing outdoor positioning performance according to Quuppa's recommendations. Two-dimensional (2D) tracking mode is chosen in the RTLS because the accuracy in the height direction is lower than in the $x$ and $y$ directions.

\subsection{Proximity detection and alert generation testing}

Five anonymous volunteer workers joined the test. They were asked to attach tags on the sides of their hardhats. In addition, three tags were attached on the body of an equipment as shown in Figure 4. Tapes are used to reinforce the attachment of all the tags. In addition, the workers were asked to wear wristbands for generating vibro-tactile alerts [8]. The Workers and the equipment operators were asked to complete their tasks according to their schedule. The system recorded the data and detected proximities. After applying the RTLS system on daily activities, the system could show the position of workers and equipment as shown in Figure 5. In this figure, the proximity between Equipment- 2 and Worker-D are indicated. The system monitored the distance between workers and equipment not belonging to the same group. Whenever the distance is less than $2 \mathrm{~m}$, the ID of the equipment and the worker are shown on the user interface. An initial test was done for alert generation. The generated vibro-tactile alerts were received by the respective workers within $1 \mathrm{~s}$.

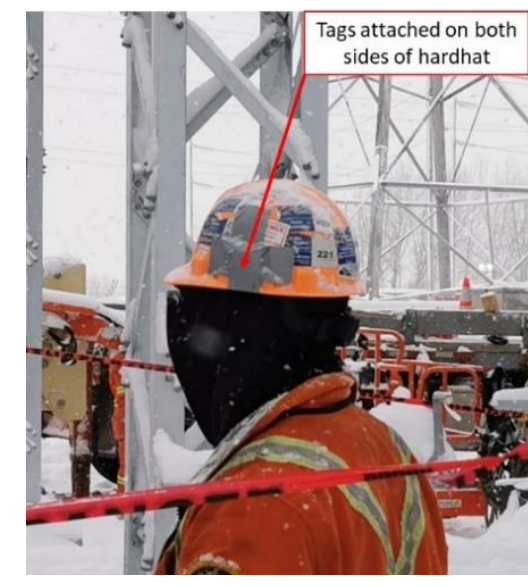

(a)

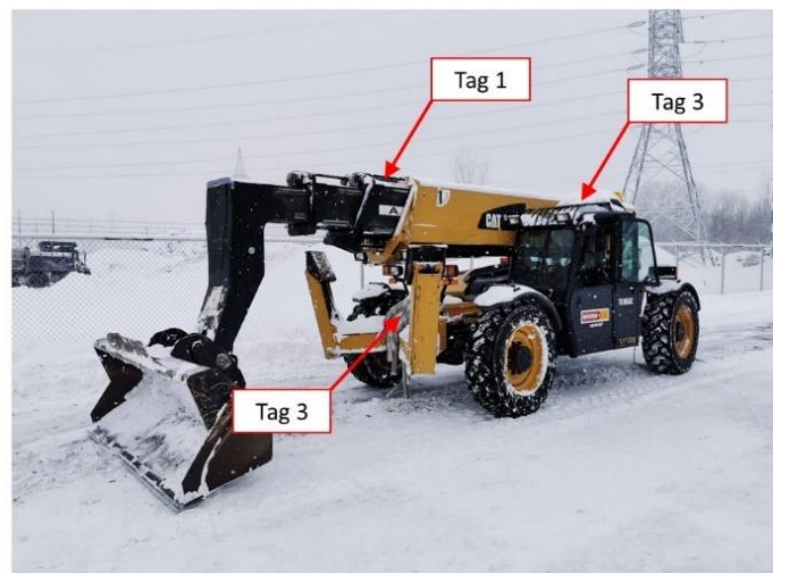

(b)

Figure 9. (a) Tags on Hardhat; (b) Tags on Telehandler 


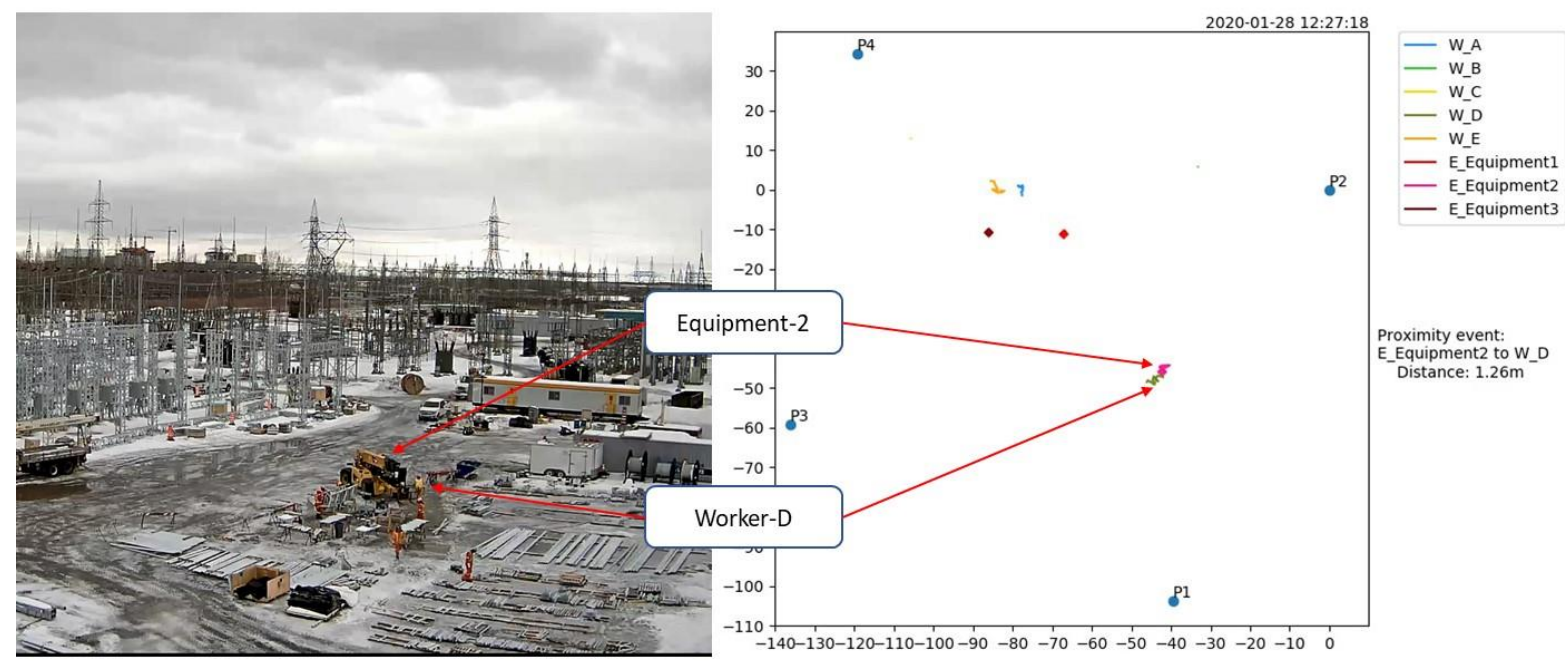

Figure 10. Monitoring Position of Equipment and Workers

\section{Conclusions and future works}

The paper proposed a method to generate near real-time alerts to both workers and equipment operators by applying a wireless scheme of BLE RTLS on a construction site. The method estimated the poses and velocity of objects by considering the positions of multiple related tags during short periods. The estimated information of the objects is used to detect risky proximities. A test on a construction site was conducted. The results were compared with the ground truth data. The BLE RTLS is able to provide accurate positioning in a large site. The method is able to monitor the position of equipment and workers and detect unsafe proximities. Furthermore, vibro-tactile alerts can be generated by the system and are perceived immediately within $1 \mathrm{~s}$ by the workers wearing a wristband.

Future work will focus on three aspects. First, more rules will be added to the method when estimating the pose and motion state of objects in order to reduce false alerts. Second, more tests will be performed during construction activities to test the feasibility of applying the proposed proximity warning system in the long term. Third, by considering feedbacks from workers, methods for improving the usability of the system will be developed.

\section{References}

[1] Zhu, Z., Park, M., Koch, C., Soltani, M., Hammad, A., \& Davari, K. (2016). Predicting movements of onsite workers and mobile equipment for enhancing construction site safety. Automation in Construction, 68, 95-101. https://doi.org/10.1016/j.autcon.2016.04.009

[2] Chae, S., \& Yoshida, T. (2010). Application of RFID technology to prevention of collision accident with heavy equipment. Automation in Construction, 19(3), 368-374. https://doi.org/10.1016/j.autcon.2009.12.008

[3] Teizer, J. (2015). Wearable, wireless identification sensing platform: Self-monitoring alert and reporting technology for hazard avoidance and training (SmartHat). Journal of Information Technology in Construction (ITcon), 20(19), 295-312. https://www.itcon.org/2015/19

[4] Ruiz, A. R. J., \& Granja, F. S. (2017). Comparing ubisense, bespoon, and decawave uwb location systems: Indoor performance analysis. IEEE Transactions on Instrumentation and Measurement, 66(8), 2106-2117. https://doi.org/10.1109/TIM.2017.2681398

[5] Siddiqui, H., Vahdatikhaki, F., \& Hammad, A. (2019). Case study on application of wireless ultra-wideband technology for tracking equipment on a congested site. Journal of Information Technology in Construction, 24, 167-187. https://www.itcon.org/2019/10

[6] Quuppa. (2019). Quuppa academy training. Unpublished manuscript.

[7] Saket, B., Prasojo, C., Huang, Y., \& Zhao, S. (2013). Designing an effective vibration-based notification interface for mobile phones. The Proceedings of the 2013 Conference on Computer Supported Cooperative Work, pp. 149-1504. https://doi.org/10.1145/2441776.2441946

[8] Blueup. (2019). Blueup BlueBeacon SafeX. https://www.blueupbeacons.com/index.php?page=safex 